Sādhanā, Vol. 19, Part 5, October 1994, pp. 833-850. (C) Printed in India.

\title{
A novel enthalpy formulation for multidimensional solidification and melting of a pure substance
}

\author{
A W DATE \\ Mechanical Engineering Department, Indian Institute of Technology, \\ Powai, Bombay 400076, India
}

\begin{abstract}
This paper presents a new finite-difference formulation of the multidimensional phase change problems involving unique phase change temperature. The solutions obtained with this formulation show that the problem of "waviness" of the temperature histories encountered with the conventional enthalpy formulation is now removed. The formulation derived provides a simple method for "local" tracking of the interface using the enthalpy variable in a novel way. During the solution of the finite-difference equations, the present formulation obviates the need for "book-keeping" of the phase-change nodes, and hence allows solution of the equations by tridiagonal matrix algorithm. It is argued that the benefits of enthalpy formulation can be extended to phase-change problems involving convection by solving the equations of motion on non-staggered grid.
\end{abstract}

Keywords. Enthalpy formulation; multidimensional phase-change problems; finite-difference method.

\section{Introduction}

\subsection{The problem considered}

There is hardly any engineering product that, during its manufacture, does not undergo a process of solidification and melting at some stage: Engineering processes such as casting, welding, surface alloying, dip forming, crystallisation etc. involve phase-change. The process of freezing and thawing is of interest in preservation of foods. The phenomenon of "permafrost" is concerned with changes in load-bearing capacity of soils in very cold environments. The principle of latent heat transfer is used in the development of compact thermal energy storage devices that enable storage and retrieval of energy at nearly constant temperature.

The phenomenon of solidification or melting is brought about by a process of latent heat $(\lambda)$ transfer at the interface between the solid and the liquid phases. For a pure substance, throughout this process, the temperature $T_{m}$ of the interface remains constant. Both $\lambda$ and $T_{m}$ are properties of a pure substance. Within each of the single 
phase, the heat transfer is essentially governed by the process of unsteady conduction, although under certain circumstances, convection may also occur in the liquid phase under the action of body (e.g. buoyancy) or surface (e.g. surface tension) forces.

There are two approaches to solving the phase change problems:

(i) the variable domain formulation;

(ii) the fixed domain formulation.

In the first approach, which has several variants, two energy equations in their conventional form are solved in the solid and the liquid phases with temperatures $T_{s}$ and $T_{l}$ as the dependent variables respectively. In addition to the domain boundary and initial conditions, the following two conditions are imposed at the interface:

$$
\begin{aligned}
& T_{s}=T_{l}=T_{m}, \\
& \left.K \frac{\partial T_{s}}{\partial \mathbf{n}}\right|_{i}-\left.K \frac{\partial T_{l}}{\partial \mathbf{n}}\right|_{i}=\rho \lambda v_{i},
\end{aligned}
$$

where $\mathrm{n}$ is a vector normal to the interface and $v_{i}$ is the instantaneous velocity of the interface in the direction of the normal.

Since the method requires continuous tracking of the interface, the physical coordinates are usually normalised with respect to this location. The governing energy equations are then freshly derived in the transformed coordinate system in which the interface is immobilised, although in the physical coordinate system the volumes occupied by each of the single phases change with time (and, hence, the designation of variable domain formulation). In multidimensional problems, this transformation involves laborious algebra that gives rise to psuedo-convection terms and crossderivatives (see, for example, Saitoh 1978), the former may even given rise to numerical instabilities under certain circumstances. The finite difference implementation of the variable domain formulation can be carried out through several variants that are described by Basu \& Date (1988); not all of which are amenable to easy extension to multidimensional problems.

This paper is concerned with the second type of formulation, namely the fixed domain or the enthalpy formulation, which treats the total enthalpy $H$, rather than the temperature $T$, as the main dependent variable in the energy equation. Now since enthalpy is a conserved property; the energy equations for both phases can be written in terms of a single equation, viz:

$$
\frac{\partial}{\partial t}(\rho H)+\operatorname{div}(\rho \mathbf{u} H)=\operatorname{div}(K \operatorname{grad} T)
$$

where $\mathbf{u}$ is the velocity vector which may be finite in the liquid phase.

Incidentally, it can be shown that (3) already satisfies the interface flux condition (2). As such, the equation applies to the entire domain of interest and the interface need not be tracked during the differential formulation of the phase-change problem. Hence, this formulation is also known as the fixed domain formulation. Note, however, that (3) contains two dependent variables, $H$ and $T$, and a set of auxiliary relations (also known as equations of state) between them must be specified.

In several applications, the domain boundaries are often of complex shape. Also because of the asymmetries of the boundary shapes, and thermal boundary conditions, and because of the presence of convection, the interface, during its evolution, can 
assume complex shapes. It has become increasingly apparent that such complexities (Wilson et al 1978; Sparrow et al 1988, pp. 747-86) can best be handled by the enthalpy formulation; rather than the variable domain formulation. This is particularly so if it is of interest to develop generalised computer codes for the general problem of solidification and melting.

Computationally speaking, it is relatively simple to implement the enthalpy formulation via discretised equations when the substance is impure. For, in this case, the latent heat transfer takes place over a range of temperatures that demarcate what is known as the "mushy" region. The physical and transport properties of this region must however be known, or modelled. For a pure substance, however, the phasechange takes place at a unique temperature and it was shown by Voller et al (1979), that unless special procedures are adopted, the predicted temperature and heat flux histories, as well as the interface movement, are unrealistic. Recently, Voller (1990) has reviewed several implicit procedures using the enthalpy formulation. Date (1991, 1992) has also reviewed some of the earlier methods and identified their shortcomings.

Recently, Date (1992) has presented an enthalpy formulation that eliminates the problem of prediction of unrealistic temperature histories, allows use of an efficient line-by-line numerical integration algorithm and is applicable to multidimensional problems. The purpose of this paper is to present Date's formulation giving further details than those given in Date (1992).

\subsection{Outline of the paper}

The paper is divided into five sections. Section 2 describes Date's (1992) formulation in one and two dimensions, $\$ 3$ deals with phase-change problems involving convection. Here it is argued that since the interface can assume arbitrary shape, solution of equations of motion on non-staggered grids offers considerably more computational convenience than the use of staggered grids. A few illustrative solutions to the phase-change problems are presented in $\S 4$. Finally conclusions are reported in $\S 5$.

\section{Enthalpy formulation of Date (1992)}

\subsection{One-dimensional problems}

For the purposes of discussion, we consider one-dimensional heat transfer without bulk convection; further assuming uniform properties. Equation (3) can then be written as:

$$
\rho \frac{\partial H}{\partial t}=k \frac{\partial^{2} T}{\partial X^{2}}
$$

In order to solve the above equation, $H$ must be replaced by $T$ or vice versa. This is done via the equations of state which provide the $\mathrm{H}-\mathrm{T}$ relationship. Figure 1 shows this relationship which is only piece-wise continuous. Mathematically, the relationship can be written in two ways as shown in table 1 . Most of the previous authors have preferred the $H=f(T)$ relationship; we prefer the $T=f(H)$ relationship following Shamsunder \& Sparrow (1975).

It is convenient to define the following dimensionless variables:

$$
\phi=\left(H-H_{s}\right) / \lambda,
$$




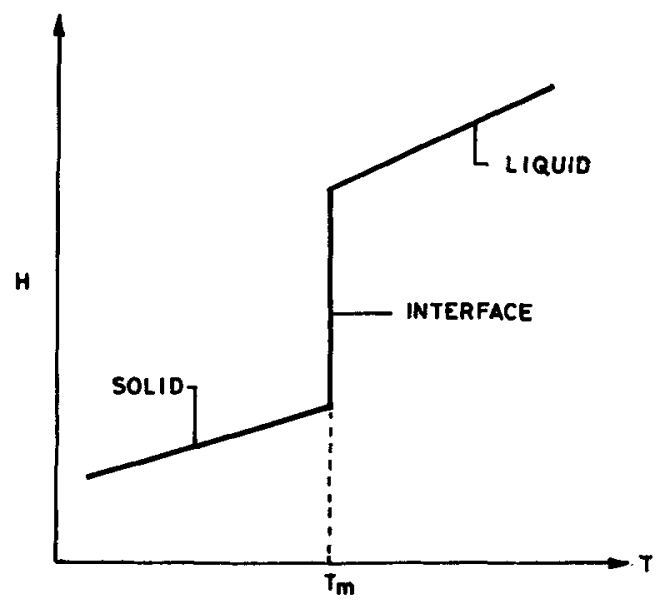

$$
\begin{aligned}
\theta & =C_{p}\left(T-T_{m}\right) / \lambda, \\
X & =x / L, \\
\tau & =\alpha t / L^{2} .
\end{aligned}
$$

Figure 1. $H-T$ relationship for a pure substance.

In the new variables, (4) reads as:

$$
\frac{\partial \phi}{\partial \tau}=\frac{\partial^{2} \theta}{\partial X^{2}}
$$

and the $T=f(H)$ relationship reads as:

$$
\begin{array}{lll}
\theta=\phi ; & \theta \leqslant 0 & \text { (solid), } \\
\theta=0 ; & 0 \leqslant \phi \leqslant 1 & \text { (interface), } \\
\theta=\phi-1 ; & \phi \geqslant 1 & \text { (liquid). }
\end{array}
$$

Relationships (10) to (12) can be generalised as:

$$
\theta=\phi+\phi^{\prime}
$$

Table 1. $H-T$ relationship.

\begin{tabular}{lll}
\hline & \multicolumn{1}{c}{$H=f(T)$} & \multicolumn{1}{c}{$T=f(H)$} \\
\hline Solid & $H=C_{p} T ; T \leqslant T_{m}$ & $T=H / C_{p} ; H \leqslant H_{s}$ \\
Interface & $H=C_{p} T_{m}+H_{p s}(t) ; T=T_{m}$ & $T=T_{m} ; H_{s} \leqslant H \leqslant H_{1}$ \\
Liquid & $H=C_{p} T+\lambda ; T \geqslant T_{m}$ & $T=(H-\lambda) / C_{p} ; H \geqslant H_{1}$ \\
& $\int^{t+t^{\prime}} \frac{\mathrm{d} H_{p s} \mathrm{~d} t=H_{1}-H_{s}=\lambda}{\mathrm{d} t}$ & \\
& where $t^{\prime}=$ time for latent & \\
& heat transfer
\end{tabular}


where

$$
\phi^{\prime}=0.5\{|1-\phi|-|\phi|-1\} \text {. }
$$

Equation (14) ensures that $\phi^{\prime}=0$ in solid, $\phi^{\prime}=-\phi$ at the interface and $\phi^{\prime}=-1$ in liquid.

Now (9) can be finite-differenced via control-volume analysis as:

where

$$
\phi_{j}^{n}=\phi_{j}^{o}=S\left(\theta_{j+1}^{n}-2 \theta_{j}^{n}+\theta_{j-1}^{n}\right)
$$

$$
S=\Delta \tau / \Delta X^{2}
$$

and $j$ identifies the grid node, whereas superscripts $n$ and $o$ identify the new and the old values. In deriving (15), uniform grid spacing $\Delta X$ is assumed.

Equation (13) can now be used to replace $\theta^{\prime} s$ in (15), so that:

$$
\phi_{j}^{n}(1+2 S)=S\left(\phi_{j+1}^{n}+\phi_{j-1}^{n}\right)+S\left(\phi_{j+1}^{\prime n}-2 \phi_{j}^{\prime n}+\phi_{j-1}^{\prime n}\right)+\phi_{j}^{0} .
$$

In the above equation, $\phi^{\prime}$ values lag behind the $\phi$ values by one iteration. As such the $\phi^{\prime}$ terms (along with $\phi_{j}^{a}$ ) can be treated as sources. Equation (17) is then unconditionally stable according to the Scarborough criterion, and can be solved by the point-by-point Gauss-Seidel scheme or by the line-by-line Tridiagonal Matrix algorithm (TDMA). Further, it is not necessary to carry out "book-keeping" of the nodes which are in solid, liquid and phase-change states.

The formulation is thus an improvement over that of Shamsunder \& Sparrow (1975) who did not generalise the $T=f(H)$ relationship in the manner of (13) and (14) and therefore had to resort to node "book-keeping" which necessitates the use of only point-by-point integration procedure. Basu \& Date (1987) have however shown that application of TDMA results in much faster convergence than the point-by-point procedure particularly when fine mesh size is used.

This formulation however suffers from one drawback. It will be recognised that $0<\phi<1$ at the phase-change node. As such, throughout the period of transition of the interface through the control-volume surrounding the phase-change node $\phi^{\prime}=-\phi$, and therefore the nodal value of the phase-change node remains stationary at $\theta=\phi+\phi^{\prime}=0$. As a result, the predicted temperature histories demonstrate a step-like or a wavy pattern (Shamsunder 1978, pp. 165-83; Voller et al 1979).

One way in which this peculiar behaviour can be eliminated is to use a fine mesh (or small $\Delta X$ ); so that the time period over which $\theta$ remains stationary at zero is minimised, and the essentially way solutions appear to be smooth and accurate. This measure was adopted by Shamsunder (1978, pp. 165-83), but it exacts penalty in computer time which can partly be compensated by the application of TDMA, as described above.

To prevent $\theta$ from remaining stationary at zero at the phase change node, it is necessary to rewrite the generalised $T=f(H)$ relationship as:

$$
\theta=\phi+\phi^{\prime \prime}
$$

where

$$
\phi^{\prime \prime}=\phi^{\prime}+\theta_{p c}
$$

Here $\theta_{p c}$ denotes the nodal value of the temperature at the phase-change node; it equals zero at the single phase nodes. With the replacement suggested by $(18),(15)$ 
can now be written as:

$$
\phi_{j}^{n}(1+2 S)=S\left(\phi_{j+1}^{n}+\phi_{j-1}^{n}\right)+S\left(\phi_{+1}^{\prime \prime n}-2 \phi_{j}^{\prime n}+\phi_{j-1}^{\prime \prime n}\right)+\phi_{j}^{o} .
$$

The above equation is same as (17), except that $\phi^{\prime}$ is replaced by $\phi^{\prime \prime}$. The $\phi^{\prime \prime}$ values again lag behind the $\phi$ values by one iteration. Further, however, it becomes necessary to determine the value of $\theta_{p c}$ at the phase-change node.

2.1a Determination of $\theta_{p c}$ : Consider figure 2, where the phase change node $j$ is shown along with nodes $j-1$ and $j+1$ which are in single phase. At the time instant considered, let the interface be located at a distance $\Delta X_{i}$ to the east of the nodal position $X_{j}$. Now since the value of $\theta$ at the interface is zero, one may linearly interpolate $\theta_{p c, j}$ to read as

$$
\theta_{p c, j}=\left[\frac{\Delta X_{i}}{\Delta X_{i}+\Delta X}\right] \theta_{j-1} .
$$

Similarly, if the interface is to the west of node $j$ then:

$$
\theta_{p c, j}=\left[\frac{\left|\Delta X_{i}\right|}{\left|\Delta X_{i}\right|+\Delta X}\right] \theta_{j+1},
$$

where

$$
\begin{aligned}
\Delta X_{i} & =X_{i}-X_{j} \\
& =\left(0.5-\phi_{j}\right) \Delta x \\
& =\left(0.5+\phi_{j}^{\prime \prime}\right) \Delta X .
\end{aligned}
$$

Equations (21) and (22) can now be generalised as:

where

$$
\theta_{p c, j}=0 \cdot 5\left[(A+|A|) \theta_{j-1}-(A-|A|) \theta_{j+1}\right] F,
$$

and

$$
A=\frac{0.5+\phi_{j}^{\prime \prime}}{1+\left|0.5+\phi_{j}^{\prime \prime}\right|}
$$

$$
F=-\frac{\left(1+\phi_{j}^{\prime}\right) \phi_{j}^{\prime}}{\left(1-\phi_{j}\right) \phi_{j}}
$$

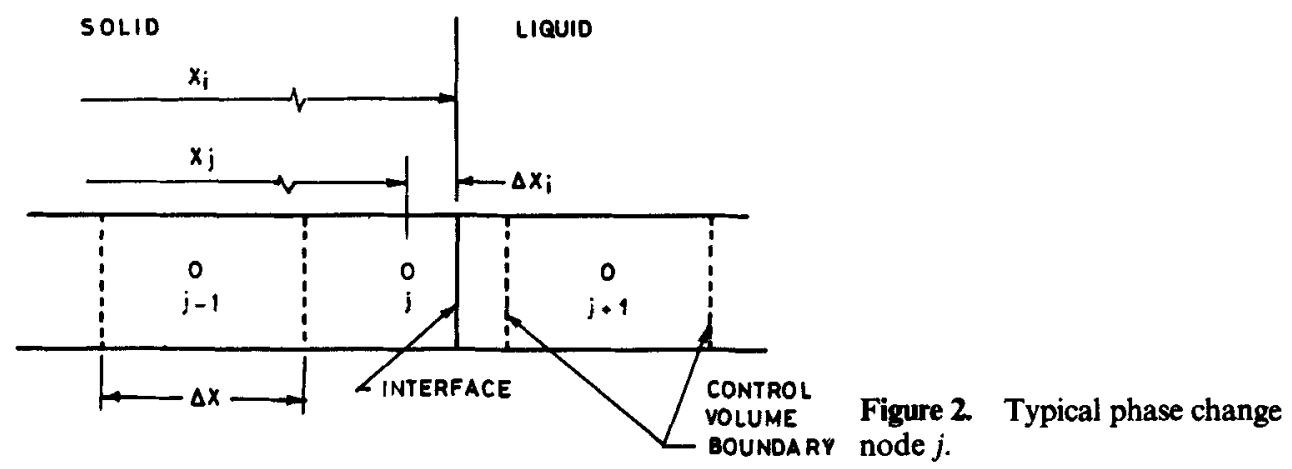


2.1b Some pertinent comments: (i) At the phase change node, $\phi_{j} \Delta X$ represents the volume occupied by the liquid.

(ii) The location of the interface is identified with $\theta=\phi+\phi^{\prime \prime}=0$. As such, $\Delta X_{i}=$ $0.5 \Delta X-\phi_{j} \Delta X=\left(0.5+\phi_{j}^{\prime \prime}\right) \Delta X$, as shown by (23).

(iii) Equation (24) ensures that when $\Delta X_{i}$ is positive, (21) is used to determine the nodal value of temperature at the phase-change node. When. $\Delta X_{i}$ is negative, (22) is operational.

(iv) The factor $F$ given by (26) equals zero at the single phase nodes, but it equals one at the phase-change node [see (14)]. This ensures that $\theta_{p c}=0$ at single phase node but is finite at the phase change node.

(v) Since $\theta_{p c, j}=0$ at the single-phase nodes $\phi_{j}^{\prime \prime}=\phi_{j}^{\prime}$ at these nodes, that is $\phi_{j}^{\prime \prime}=0$ in solid and $\phi_{j}^{\prime \prime}=-1$ in liquid. As such the location of $X_{i}$ (or the volume occupied by the solid) can be calculated from:

$$
X_{i}=\sum_{j=1}^{N}\left(1+\phi_{j}^{\prime \prime}\right) \Delta X
$$

where $N$ denotes the total number of nodes in the domain.

(vi) Both $\phi^{\prime}$ and $\theta_{p c}$ are calculated in such a way that it is not necessary to carry out "book-keeping" of the single-phase and phase-change nodes, and hence (20) can be solved by TDMA.

2.1c The solution procedure: The following steps are incorporated in the solution procedure:

(1) Specify initial values of temperature $\theta_{\text {in }}$ at all nodes.

(2) Hence, evaluate $\phi_{i n}$ and $\phi_{i n}^{\prime}$ at all nodes using (10) or (12) as appropriate, and (14). Set $\phi_{i n}^{\prime \prime}=\phi_{i n}^{\prime}$.

(3) Perform one iteration of (20) for an arbitrarily chosen time step $\Delta \tau$ to yield new values of $\phi$.

(4) Evaluate $\phi^{\prime}$ from (14) and $\theta_{p c}$ from (24) at all nodes using the just calculated values of $\phi$. Hence form $\phi^{\prime \prime}=\phi^{\prime}+\theta_{p c}$ at all nodes.

(5) Return to step (3) until $\phi$ has converged between successive iterations.

(6) Calculate $\theta=\phi+\phi^{\prime \prime}$ at all nodes and evaluate $X_{i}$ from (27) if desired.

(7) Set $\phi^{o}=\phi^{n}$, and return to step 3 to calculate the next time step.

The above strongly implicit procedure is stable for any value of the chosen time step $\Delta \tau$, and grid spacing $\Delta X$. Usually coarse grids (say 5 to 7 nodes) suffice. However, for high Stefan numbers, that is for high velocity of interface movement, finer grids (11 to 13 nodes) may be required to achieve good accuracy (Date 1992).

\subsection{Two-dimensional problems}

The dimensionless governing equation for such problems is given by

$$
\frac{\partial \phi}{\partial \tau}=\frac{\partial^{2} \theta}{\partial X^{2}}+\frac{\partial^{2} \theta}{\partial Y^{2}}
$$


and the finite-difference analogue is given by:

where

$$
\begin{aligned}
\phi_{k, j}^{n}\left(1+2 S_{x}+2 S_{y}\right)= & S_{x}\left(\phi_{k+1, j}^{n}+\phi_{k-1, j}^{n}\right) \\
& +S_{y}\left(\phi_{k, j+1}^{n}+\phi_{k, j-1}^{n}\right) \\
& +S_{x}\left(\phi_{k+1, j}^{\prime \prime n}-2 \phi_{k, j}^{\prime \prime n}+\phi_{k-1, j}^{\prime n}\right) \\
& +S_{y}\left(\phi_{k, j+1}^{\prime \prime n}-2 \phi_{k, j}^{\prime \prime n}+\phi_{k, j+1}^{\prime \prime n}\right) \\
& +\phi_{k, j}^{o},
\end{aligned}
$$

$$
\begin{aligned}
& S_{x}=\Delta \tau / \Delta X^{2}, \\
& S_{y}=\Delta \tau / \Delta Y^{2} .
\end{aligned}
$$

2.2a Determination of $\theta_{p c}$ : In order to evaluate $\phi_{k j}^{\prime \prime}$, it becomes necessary to estimate $\theta_{p c, k j}$ at all phase-change nodes. Note that, in a multi-dimensional problem, several nodes may undergo phase change simultaneously. The procedure adopted for evaluation of $\theta_{p c, k j}$ is as follows:

where

$$
\theta_{p c, k j}=0.5\left(\theta_{p c x}+\theta_{p c y}\right) F
$$

$$
F=-\left\{\left(1+\phi_{k j}^{\prime}\right) \phi_{k j}^{\prime}\right\} /\left\{\left(1-\phi_{k j}\right) \phi_{k j}\right\}
$$

Now the $X$ and $Y$ components of $\theta_{p c}$ are evaluated as follows:

where

$$
\theta_{p c x}=0 \cdot 5\left[\left(\frac{B x}{|B x|}+1\right) \theta_{p c x_{1}}-\left(\frac{B x}{|B x|}-1\right) \theta_{p c x_{2}}\right]
$$

and

$$
\begin{aligned}
B x & =|A x|-0 \cdot 5 ; \\
A x & =\Delta X_{i} /\left\{\left|\Delta X_{i}\right|+\Delta X\right\}, \\
\theta_{p c x_{1}} & =0 \cdot 5\left(\theta_{k-l, j}+\theta_{k+l, j}\right) ;
\end{aligned}
$$

$$
\theta_{p c x_{2}}=0 \cdot 5\left[(A x+|A x|) \theta_{k-l, j}-(A x-|A x|) \theta_{k+l, j}\right] \text {. }
$$

Similarly

$$
\theta_{p c y}=0 \cdot 5\left[\left(\frac{B y}{|B y|}+1\right) \theta_{p c y_{1}}-\left(\frac{B y}{|B y|}-1\right) \theta_{p c y_{2}}\right] \text {, }
$$

where

$$
\begin{aligned}
B y & =|A y|-0.5, \\
A y & =\Delta Y_{i} /\left\{\left|\Delta Y_{i}\right|+\Delta Y\right\} \\
\theta_{p c y_{1}} & =0.5\left(\theta_{k, j-1}+\theta_{k, j+1}\right),
\end{aligned}
$$

and

$$
\theta_{p c y z}=0 \cdot 5\left[(A y+|A y|) \theta_{k, j-1}-(A y-|A y|) \theta_{k, j+1}\right] .
$$

Here $\Delta X_{i}$ and $\Delta Y_{i}$ represent the location of the interface along $X$ and $Y$ axes respectively (see figure 3 ). Note that in a general phase-change problem, the interface may occupy the phase-change control volume in any of the six ways shown in figure 3. 


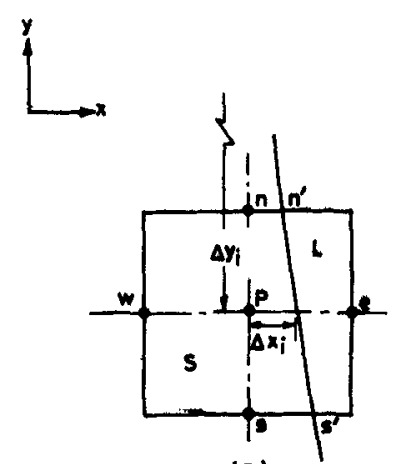

(a)

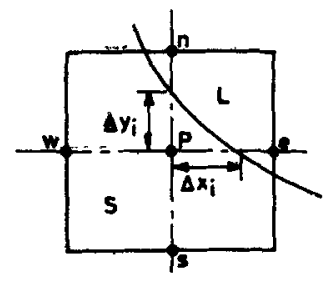

(c)

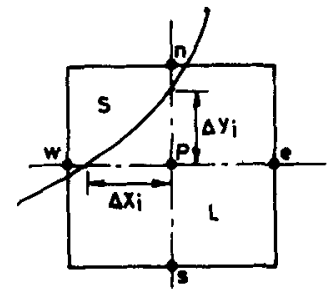

(e)

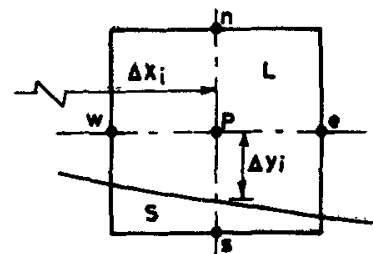

(b)

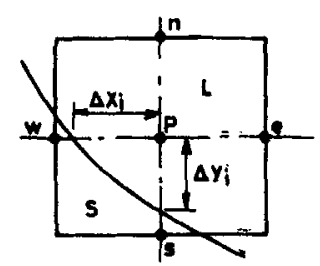

(d)

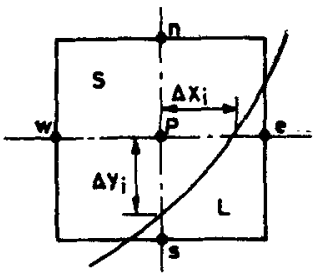

(f)
Figure 3. Different interface positions.

Equations (33) and (34) show that when $\left|\Delta X_{i}\right| \geqslant \Delta X$ or $\left|\Delta Y_{i}\right| \geqslant \Delta Y$, then $\theta_{p c x}=\theta_{p c x 1}$ and $\theta_{p c y}=\theta_{p c y 1}$; when $\left|\Delta X_{i}\right| \leqslant \Delta X$ and $\left|\Delta Y_{i}\right| \leqslant \Delta Y, \theta_{p c x}=\theta_{p c x \times 2}$ and $\theta_{p c y}=\theta_{p c y 2}$. Thus, one-sided interpolation for $\theta_{p c}$ is carried out only when the interface lies between the phase-change node and its immediate neighbouring node.

2.2b Determination of $\Delta X_{i}$ and $\Delta Y_{i}$ : It is clear from figure 3 that the expressions for $\Delta X_{i}$ and $\Delta Y_{i}$ will depend on the type of intersection of the interface with the control volume. Further, it must be possible to determine the type of intersection itself. Both these are accomplished by using $\phi^{\prime}$ and $\phi^{\prime \prime}$ variables. Here expressions for Type (a) and Type (c) intersections will be devised; for other types, the appropriate expressions are given in the appendix $A$.

Type (a) intersection is thus identified by:

$$
\begin{aligned}
& \phi_{k+1, j}^{\prime}=-1 \\
& \phi_{k-1, j}^{\prime}=0 .
\end{aligned}
$$

Further, area $n n^{\prime} s s^{\prime}=\left(0.5+\phi_{k j}^{\prime \prime}\right) \Delta X \Delta Y$, or

$$
\Delta X_{i}=\left(0.5+\phi_{k j}^{\prime \prime}\right) \Delta X
$$


In order to determine $\Delta Y_{i}$, the procedure adopted is as follows:

Now

$$
\Delta Y_{i}=-\left.\frac{\partial \Delta Y_{i}}{\partial X}\right|_{k j} \Delta X_{i}-\left.\frac{\partial^{2} \Delta Y_{i}}{\partial X^{2}}\right|_{k j} \frac{\Delta X_{i}^{2}}{2}+\text { neglected terms }
$$

and

$$
\frac{\partial \Delta Y_{i}}{\partial X}=1 /\left(\frac{\partial \Delta X_{i}}{\partial Y}\right)=-1 /\left(\left.\frac{\partial \phi}{\partial Y}\right|_{k j}\right) \Delta X=1 /\left(\left.\frac{\partial \phi^{\prime}}{\partial Y}\right|_{k j}\right) \Delta X,
$$

Thus,

$$
\frac{\partial^{2} \Delta Y_{i}}{\partial X^{2}}=-\left.\left\{\frac{\partial^{2} \Delta X_{i}}{\partial X \partial Y} /\left(\frac{\partial \Delta X_{i}}{\partial Y}\right)^{2}\right\}\right|_{k j}=-\left\{\left.\frac{\partial^{2} \phi^{\prime}}{\partial X \partial Y}\right|_{k j} /\left(\left.\frac{\partial \phi^{\prime}}{\partial Y}\right|_{k j}\right)^{2} \Delta X\right\}
$$

where

$$
\Delta Y_{i}=-C_{1}+\left.\frac{C_{1}^{2} \Delta X}{2} \frac{\partial^{2} \phi^{\prime}}{\partial X \partial Y}\right|_{k j}
$$

$$
C_{1}=\left(0 \cdot 5+\phi_{k j}^{\prime \prime}\right) /\left.\left(\partial \phi^{\prime} / \partial Y\right)\right|_{k j}
$$

The derivatives of $\phi^{\prime}$ are evaluated by central difference. Equation ( $35 \mathrm{~b}$ ) shows that when the interface is parallel to the $Y$-axis (as in a one-dimensional problem) $\partial \phi^{\prime} / \partial Y=0$ and $\Delta Y_{i} \rightarrow \infty$ as would be expected; and (35a) is the same as (23).

Type $(c)$ intersection is identified by:

Further,

$$
\phi_{k-1, j}^{\prime}=\phi_{k, j-1}^{\prime}=0 \text {. }
$$

where

$$
\Delta Y_{i}=\left(Y_{i}-Y_{n}\right)+0 \cdot 5 \Delta Y,
$$

$$
\begin{aligned}
Y_{i}-Y_{n} & =-\left.\frac{\partial \Delta Y_{i}}{\partial X}\right|_{n}-\left.\frac{\partial^{2} \Delta Y_{i}}{\partial X^{2}}\right|_{n} \frac{\Delta X_{i n}^{2}}{2}+\ldots \\
\Delta X_{i n} & =X_{i n}-X_{n}=\left(0 \cdot 5+\phi_{n}^{\prime \prime}\right) \Delta X \\
\phi_{n}^{\prime \prime} & =\phi_{k j}^{\prime \prime}+\phi_{k, j+1}^{\prime \prime}+\frac{1}{8}\left[\phi_{k-1, j+1}^{\prime \prime}+\phi_{k-1, j}^{\prime \prime}+\phi_{k+1, j+1}^{\prime \prime}+\phi_{k+1, j}^{\prime \prime}\right] \\
\left.\frac{\partial \Delta Y_{i}}{\partial X}\right|_{n} & =1 /\left\{\left(\left.\frac{\partial \phi^{\prime}}{\partial Y}\right|_{n}\right) \Delta X\right\}
\end{aligned}
$$

and

$$
\left.\frac{\partial^{2} \Delta Y_{i}}{\partial X^{2}}\right|_{n}=-\left.\left\{\frac{\partial^{2} \phi^{\prime}}{\partial X \partial Y} /\left(\frac{\partial \phi^{\prime}}{\partial Y}\right) \Delta X\right\}\right|_{n}
$$

Then

$$
\begin{aligned}
\Delta Y_{i}=0 \cdot 5 \Delta Y & -\left(0 \cdot 5+\phi_{n}^{\prime \prime}\right) /\left\{\left.\frac{\partial \phi^{\prime}}{\partial Y}\right|_{n}\right\} \\
& +\left\{\left.\left(0 \cdot 5+\phi_{n}^{\prime \prime}\right)^{2} \frac{\partial^{2}}{\partial X} \frac{\phi^{\prime}}{\partial Y}\right|_{n} \Delta X\right\} / 2\left(\left.\frac{\partial \phi^{\prime}}{\partial Y}\right|_{n}\right)^{2}
\end{aligned}
$$


Similarly, it can be shown that:

$$
\Delta X_{i}=0.5 \Delta X-\left(0.5+\phi_{e}^{\prime \prime}\right) /\left\{\left.\frac{\partial \phi^{\prime}}{\partial X}\right|_{e}\right\}+\left\{\left.\left(0.5+\phi_{e}^{\prime \prime}\right)^{2} \frac{\partial^{2} \phi^{\prime}}{\partial X \partial Y}\right|_{e} \Delta Y\right\} / 2\left(\left.\frac{\partial \phi^{\prime}}{\partial X}\right|_{e}\right)^{2},
$$

2.2c The solution procedure: Thus once $\Delta X_{i}$ and $\Delta Y_{i}$ are determined for each phase-change node, $\theta_{p c}$ can be determined from (31) to (34). The overall solution procedure is given below:

(1) Specify initial values of temperature $\theta_{i n}$ at all nodes.

(2) Hence evaluate $\phi_{i n}$ and $\phi_{i n}^{\prime}$ and set $\phi_{i n}^{\prime \prime}=\phi_{i n}^{\prime}$.

(3) Perform one iteration of (29) using double-sweep TDMA for an arbitrarily chosen time step $\Delta \tau$ to yield new values of $\phi$. Calculate $\phi^{\prime}$ values at all nodes.

(4) Identify the type of interface intersection from $\phi^{\prime}$ distribution. Hence calculate $\Delta X_{i}$ and $\Delta Y_{i}$ from appropriate expression.

(5) Calculate $\theta_{p c, k j}$ from $\Delta X_{i}$ and $\Delta Y_{i}$.

(6) Form $\phi_{k j}^{\prime \prime}=\phi_{k j}^{\prime}+\theta_{p c, k j}$.

(7) Return to step 3 until $\phi$ has converged between successive iterations.

(8) Calculate $\theta=\phi+\phi^{\prime \prime}$ at all nodes.

(9) Set $\phi^{\circ}=\phi^{n}$ and return to step 3 to calculate at the new time step.

\subsection{Closure}

In this section, an enthalpy formulation is developed, the unique feature of which is the generalisation of the $T=f(H)$ relationship first in a continuum [(13), (14)], and then for a discretised domain [(18), (19)]. The latter is introduced to facilitate prediction of smooth temperature and heat flux hisotries without employing very fine mesh sizes.

The discretised version of the $T=f(H)$ relationship required evaluation of $\theta_{p c}$ at the phase-change nodes. This, in turn, requires location of the interface in terms of local coordinates $\Delta X_{i}$ and $\Delta Y_{i}$. The $\theta_{p c}, \Delta X_{i}$ and $\Delta Y_{i}$ are evaluated in terms of $\phi^{\prime}$ and $\phi^{\prime \prime}$ which are functions of $\phi$. This method of locating the interface is unique in the sense that the interface may locally assume arbitrary shape and may intersect a given grid-line more than once. This contrasts with the method proposed by Patel (1968) (and which is used by Lazardis 1970 \& Huang et al 1991) which attempts to predict interface coordinates $X_{i}$ and $Y_{i}$ relative to a fixed origin at all times by solving a differential equation. The present method for locating interface can also be extended to three-dimensional situations by employing appropriate Taylor-series expansion.

Further, the present method does not require "book-keeping" of the nodes in any of the operations and therefore enables use of line-by-line integration algorithm such as the TDMA.

\section{Phase-change with convection}

In several multidimensional phase-change problems, the operating or boundary conditions may be such that the liquid region experiences convection due to body or surface forces (Basu \& Date 1988), requiring solution of the Navier-Stokes 
equations to retrieve the velocity vector $\mathbf{u}$ in (3). Usually such problems are solved by using the variable domain formulation (see, for example, Beckermann \& Viskanta 1989). In such methods, since the liquid volume changes in size and shape with time, grids must be relaid at every time step with consequent requirement for interpolating the variables to the new positions assumed by the grid nodes. This is quite cumbersome. In the enthalpy formulation, since the grids remain fixed in space, such problems are avoided, although care is needed in effecting the boundary conditions on velocities at the phase-change nodes. This problem becomes particularly more complex when staggered grids are used for the velocity variables (see, for example, Gadgil \& Gobin 1984 and Voller et al 1987) since the control-volumes surrounding the different velocity components occupy different amounts of solid and liquid, and which must be properly accounted. In order to fully realise the benefits of the fixed-grid enthalpy formulation, it is necessary to solve the Navier-Stokes equations on non-staggered grids.

During the last decade this has been achieved by employing in effect the concept of artificial compressibility (Rhie \& Chow 1983) to eliminate the problem of checkerboard prediction of pressure. Recently however, Date $(1993,1994)$ has pointed out some inelegant aspects of this method and its variants and suggested a new method that involves use of an effective pressure gradient to drive the nodal velocities. This method can be easily combined with the present enthalpy formulation since the liquid volume of any node is simply calculated as $-\phi_{k j}^{\prime} \Delta X \Delta Y$ (in two dimensions). Also since $\Delta X_{i}$ and $\Delta Y_{i}$ are locally evaluated, they can be readly used to effect the no-slip boundary condition at the interface.

Complete details of present enthalpy formulation with convection are not given here for limitations of space, although these can be found in the dissertation by Pillay (1992) who has extended the formulation presented in $\$ 2$ to include the effects of non-uniform grid-spacing, property difference of the two phases and convection driven by buoyancy.

\section{Some illustrative applications}

The present enthalpy formulation has been applied to the solution of several one- and two-dimensional problems (without convection) involving temperature, heat flux and heat transfer coefficient boundary conditions and different initial conditions (Date 1992). Here, for the purposes of illustration, two 2-dimensional problems without convection and one problem with convection are considered.

Problem 1: This problem has been solved by Lazaridis (1970). Initially saturated liquid $\left(\theta_{i n}=0\right)$ is contained in a square domain of unit dimensions. At $\tau=0$, the temperature at $X=0$ and $Y=0$ boundaries is lowered to $\theta_{0}(=\mathrm{St})=-0.6405$ so that solidification commences instantly. The boundaries at $X=1$ and $Y=1$ are insulated.

Figure 4 shows the comparison of the predicted interface movement with that calculated by Lazaridis (1970). The present predictions are obtained with only five nodes in $X$ and $Y$ directions. The accuracy of predictions was checked by employing three time steps such that $S_{x}=S_{y}=0.0625,0.125,0.25$. The computed results were found to be insensitive to this variation of time steps predicting the total solidification time at $\tau=0.59$. For the largest time step the CPU time for complete solidification on CYBER $180 / 840$ computer was $2 \cdot 26 \mathrm{~s}$, and that for the smallest time step was $9 \cdot 02 \mathrm{~s}$. 


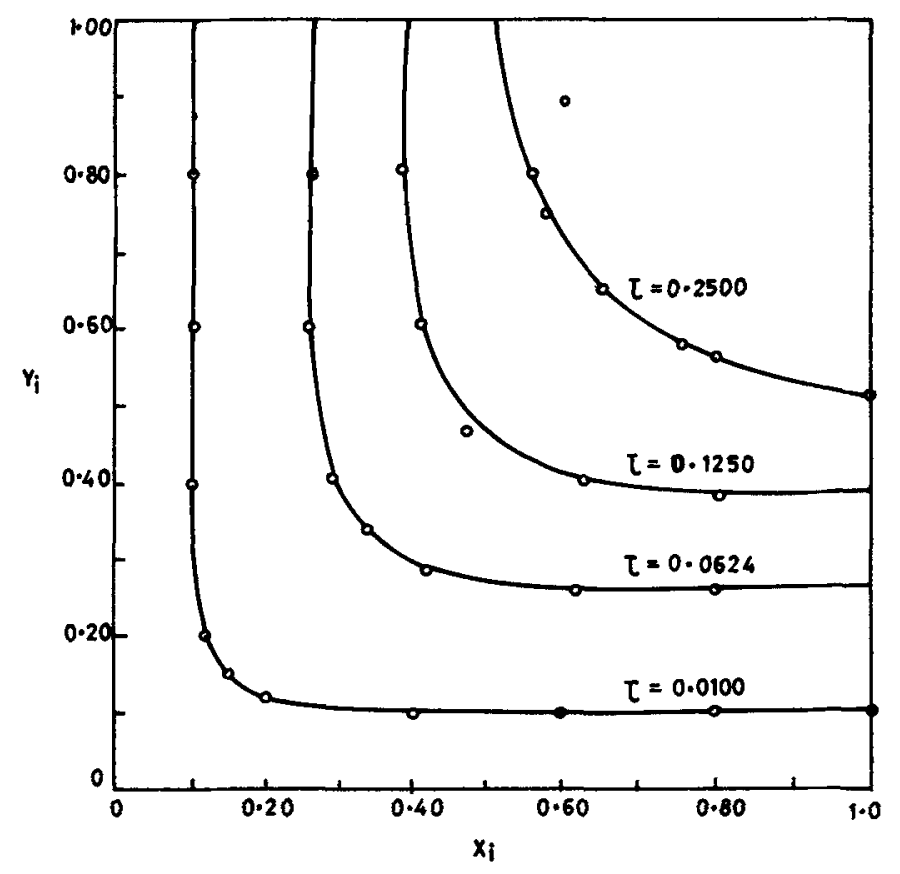

Figure 4. Evolution of interface - 2-D conduction phase change. [- Present $(\Delta X=\Delta Y=0 \cdot 2), \circ$ Lazaridis 1970.]

The special feature of this problem is that since $\theta_{o}$ is constant along $X=0$ and $Y=0$ the predicted interface is symmetric about $Y=X$ line and that at no time instant does the interface intersect a given grid line more than once.

Problem 2: In order to cause multiple intersections of the interface with a given grid-line at some instance of time, Lazaridis's problem was modified such that at $X=0$ and $Y=0, \theta_{0}$ was assumed to vary linearly as:

where

$$
\theta_{o}=-(0.25+0.75 Z)
$$

$$
Z=X \text { or } Y \text { as appropriate. }
$$

For this problem 8 nodes were used in $X$ and $Y$ directions. Figure 5 shows the interface movement as predicted by Pillay (1992). The total solidification time was predicted at $\tau=0.74$ and required CPU time of $10.4 \mathrm{~s}$ for $S_{x}=S_{y}=0.54$ and $13 \mathrm{~s}$ for $S_{x}=S_{y}=0.32$. The computed results were again found to be independent of the time step. It is seen that at small times, the interface intersects a given grid line twice.

Problem 3: Beckermann \& Viskanta (1989) performed experiments with melting of gallium. The test cell had inside dimensions of $4.76 \mathrm{~cm}$ height and width and $3.81 \mathrm{~cm}$ depth. Initially the gallium was at temperature $T_{c}<T_{m}$. At $t=0$, the right vertical face is raised to $T_{h}>T_{m}$ and maintained there while the left vertical face is maintained at $T_{c}$. All other faces are insulated.

Melting proceeds from right (i.e. $X=1)$ towards left $(X=0)$. However the process conditions are such that after sufficient time, convective heat transfer from the melt 


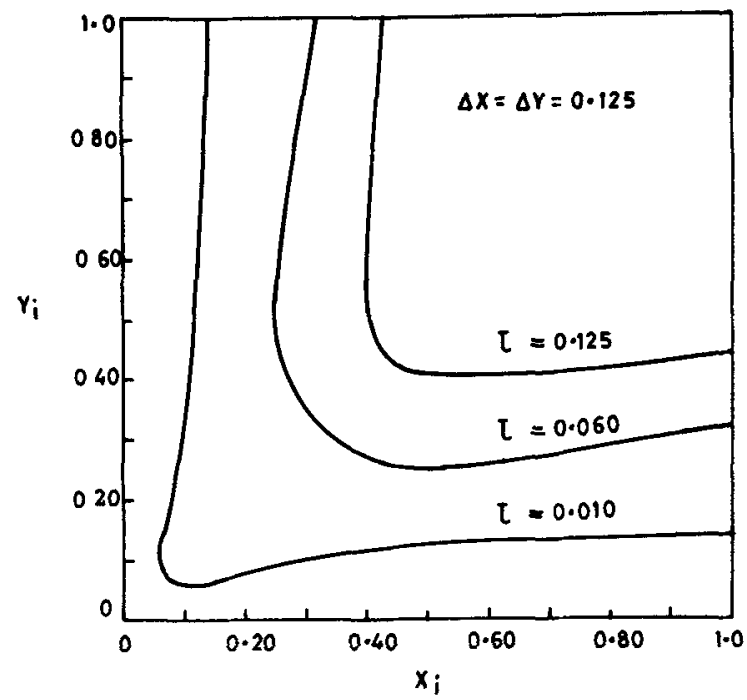

Figure 5. Evolution of interface-2-D conduction phase change.

to the interface is balanced by the heat conduction through the solid, and a steady state is reached.

Pillay (1992) performed calculations assuming the melting situation to be twodimensional neglecting all variations in the depthwise direction. The same conditions were assumed by Beckermann \& Viskanta (1989).

Figure 6 shows the comparison of predicted and experimental interface location at steady-state for three experimental conditions: $T^{*}=\left(T_{h}-T_{c}\right) /\left(T_{h}-T_{m}\right)=0.468$, $0.957,1.935$. Pillay's (1992) predictions were obtained with $10 \times 10$ fixed nodes, whereas Beckermann \& Viskanta (1989), who used a variable domain formulation, used $26 \times 42$ grid nodes in liquid and solid regions each. Pillay (1992) solved the enthalpy and momentum equations simultaneously and not through a quasi-steady approximation necessitated in the variable domain method for solving the momentum equation. Considering the coarseness of the grid, Pillay's predictions are in reasonable agreement with the experiment and the predictions of Beckermann \& Viskanta (1989). The departure of the interface shape from the vertical demonstrates the effect of buoyancy-induced circulation in the liquid region.

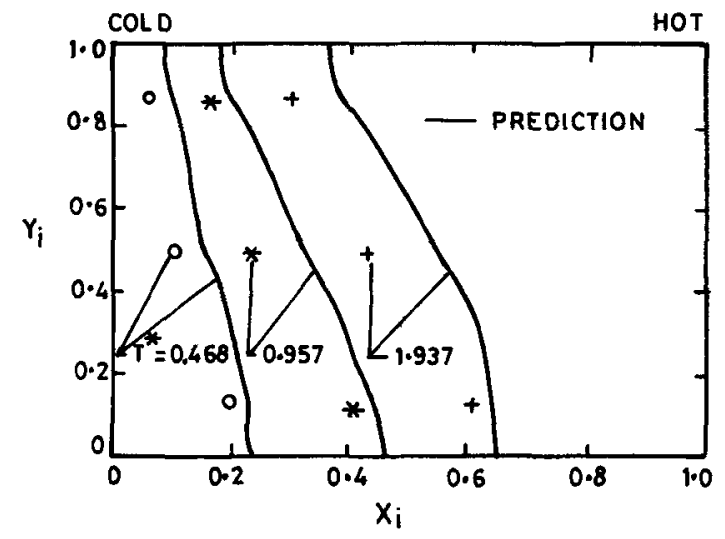

Figure 6. Interface position at steady state $-2-D$ phase change with convection. 


\section{Conclusions}

The following are the salient features of the enthalpy formulation described in the present paper.

(1) By generalising the temperature-enthalpy relationship, first in a continuum $\left(\theta=\phi+\phi^{\prime}\right)$ and then adapting it to the requirements of discretization $\left(\theta=\phi+\phi^{\prime \prime}=\right.$ $\left.\phi+\phi^{\prime}+\theta_{p c}\right)$, a calculation procedure is developed that is numerically stable and physically accurate resulting in prediction of oscillation-free, non-wavy temperature histories and interface movements. The introduction of $\theta_{p c}$ is akin to stating that phase change takes place over a range of temperatures. However, the range here is physically estimated and variable, and is not externally imposed as done by earlier researchers (see for example, Meyer 1971 or Szekely \& Themlis 1970).

(2) The $\phi^{\prime}$ and $\phi^{\prime \prime}$ variables are used to locate the interface locally, to suppress $\theta_{p c}$ to zero at single phase nodes, and to enable numerical integration of the discretised equations by line-by-line algorithm without node "book-keeping". The $\phi^{\prime}$ variable is also used to calculate the liquid portion of the control volume necessary for integrating Navier-Stokes equations when convection is present.

(3) Since the interface coordinates are calculated with respect to a local origin, rather than with respect to a fixed origin, the present method can handle interface of arbitrary shape and one that may multiply the intersect with a fixed grid line. Third-order accurate Taylor's series expansion is used to estimate the location of the interface. As such when the interface is very sharply curved, finer mesh size is required.

(4) The present method has been extended to account for differences in liquid and solid properties, non-uniform grid spacing and convection. It is suggested that solution of equations of motion on non-staggered grid (Date 1993, 1994) enhances the convenience offered by the enthalpy formulation. The method can also be extended to complex geometries, and to the use of general curvilinear coordinates.

Appendix A - Expressions for location of the interface

Refer to figure 3.

Type $(b)$ intersection $\left(\phi_{k, j-1}^{\prime}=0, \phi_{k, j+1}^{\prime}=-1\right)$

$$
\begin{aligned}
& \Delta Y_{i}=\left(0.5+\phi_{k j}^{\prime \prime}\right) \Delta Y \\
& \Delta X_{i}=-\left(0.5+\phi_{k j}^{\prime \prime}\right) /\left\{\left.\frac{\partial \phi^{\prime}}{\partial X}\right|_{k j}\right\}+\left\{\left.\left(0.5+\phi_{k j}^{\prime \prime}\right)^{2} \frac{\partial^{2} \phi^{\prime}}{\partial X \partial Y}\right|_{k j} \Delta Y\right\} /\left\{2\left(\left.\frac{\partial \phi^{\prime}}{\partial X}\right|_{k j}\right)^{2}\right\}
\end{aligned}
$$

Type (d) intersection $\left(\phi_{k, j+1}^{\prime}=\phi_{k+1, j}^{\prime}=-1\right)$

$$
\begin{aligned}
\Delta Y_{i}=-0.5 \Delta Y-(0 \cdot 5 & \left.+\phi_{s}^{\prime \prime}\right) /\left\{\left.\frac{\partial \phi^{\prime}}{\partial Y}\right|_{s}\right\} \\
& +\left\{\left.\left(0 \cdot 5+\phi_{s}^{\prime \prime}\right)^{2} \frac{\partial^{2} \phi^{\prime}}{\partial X \partial Y}\right|_{s} \Delta x\right\} /\left\{2\left(\left.\frac{\partial \phi^{\prime}}{\partial Y}\right|_{s}\right)^{2}\right\}
\end{aligned}
$$




$$
\begin{aligned}
\Delta X_{i}=-0.5 \Delta X-(0.5 & \left.+\phi_{w}^{\prime \prime}\right) /\left\{\left.\frac{\partial \phi^{\prime}}{\partial X}\right|_{w}\right\} \\
+ & \left\{\left.\left(0.5+\phi_{w}^{\prime \prime}\right)^{2} \frac{\partial^{2} \phi^{\prime}}{\partial X \partial Y}\right|_{2} \Delta Y\right\} /\left\{2\left(\frac{\partial \phi^{\prime}}{\partial X}\right)_{w}^{2}\right\}
\end{aligned}
$$

Type (e) intersection $\left(\phi_{k+1, j}^{\prime}=\phi_{k, j-1}^{\prime}=-1\right)$

$$
\begin{aligned}
\Delta Y_{i} & =\text { RHS of }(36 \mathrm{~g}), \\
\Delta X_{i} & =\text { RHS of (A4). }
\end{aligned}
$$

Type $(f)$ intersection $\left(\phi_{k, j+1}^{\prime}=\phi_{k-1, j}^{\prime}=0\right)$

$$
\begin{aligned}
\Delta Y_{i} & =\text { RHS of (A3), } \\
\Delta X_{i} & =\text { RHS of (36h). }
\end{aligned}
$$

\section{List of symbols}

$\begin{array}{ll}C_{p} & \text { specific heat; } \\ H & \text { total enthalpy; } \\ H_{p s} & \text { pseudo enthalpy; } \\ K & \text { thermal conductivity; } \\ L & \text { characteristic length; } \\ S, S_{x} & \Delta \tau / \Delta X^{2} ; \\ S_{y} & \Delta \tau / \Delta Y^{2} ; \\ S t & \text { Stefan number }\left[C_{p}\left(T_{m}-T_{\mathrm{ref}}\right) / \lambda\right] \\ t & \text { time; } \\ T & \text { temperature; } \\ v_{i} & \text { interface velocity; } \\ u & \text { velocity; } \\ X & \text { dimensionless } x \text { coordinate }(X / L) ; \\ Y & \text { dimensionless } y \text { coordinate }(Y / L) ; \\ X_{i}, Y_{i} & \text { interface coordinates; } \\ \alpha & \text { thermal diffusivity; } \\ \theta & \text { dimensionless temperature; } \\ \lambda & \text { latent heat; } \\ \rho & \text { density; } \\ \tau & \text { dimensionless time; } \\ \phi & \text { dimensionless enthalpy; } \\ \phi^{\prime} & \text { see (14); } \\ \phi^{\prime \prime} & \phi^{\prime}+\theta_{p c} \text {. }\end{array}$

\section{Subscripts}

$i \quad$ interface;

$k, j \quad$ node designation;

in initial condition; 


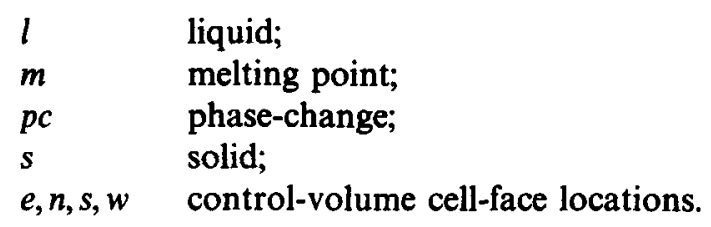

\section{Superscripts}

$\begin{array}{ll}n & \text { new value; } \\ o & \text { old value. }\end{array}$

\section{References}

Basu B, Date A W 1987 On local vs global implicitness during the solution of melting and solidification problems. Proc. 9th ISHMT Heat Mass Transfer Conference, Bangalore, India paper no. HMT-57-87

Basu B, Date A W 1988 Numerical modeling of melting and solidification problems-A review. Sădhanā 13: 169-213

Beckermann C, Viskanta R 1989 Effect of sub-cooling on natural convection melting of a pure-metal. Trans. ASME, J. Heat Transfer 3: 416-424

Date A W 1991 A strong enthalpy formulation for the Stefan problem. Int. J. Heat Mass Transfer 34: 2231-2235

Date A W 1992 Novel strongly implicit enthalpy formulation for multidimensional Stefan problems. Numer. Heat Transfer B21: 231-235

Date A W 1993 Solution of Navier-Stokes equations on non-staggered grid. Int. J. Heat Mass Transfer 36: 1913-1922

Date A W 1994 A calculation procedure for prediction of heat mass and momentum transfer in elliptic flows using non-staggered grid. Proceedings 1st ISHMT-ASME Heat Mass Transfer Conference, Bombay

Gadgil A, Gobin D 1984 Analysis of two-dimensional melting in rectangular enclosure in presence of convection. Trans. ASME, J. Heat Transfer 106: 20-26

Huang L I, Ayyaswamy P S, Cohen I M 1991 A note on interface condition in phase-change problems. Trans. ASME J. Heat Transfer 113: 244-247

Lazaridis A 1970 A numerical solution of multidimensional solidification (or melting) problems. Int. J. Heat Mass Transfer 13: 155-169

Meyer G H 1971 Multidimensional Stefan problems. SIAM J. Numer. Anal. 8: 80-96

Patel P D 1968 Interface condition in heat conduction problems with change of phase. AIAA J. 6: 2454

Pillay V R 1992 Numerical investigation of heat transfer during process of solidification \& melting with and without convection. M. Tech. Dissertation, Dept. of Mech. Eng., Indian Inst. Technol., Bombay

Rhie C M, Chow W L 1983 A numerical study of the turbulent flow past an isolated airfoil with trailing edge separation. AIAA J. 21: $1525-1532$

Saitoh T 1978 Numerical method for multidimensional freezing problems in arbitrary regions. Trans. ASME, J. Heat Transfer 100: 294-299

Shamsunder N 1978 Comparison of numerical methods for diffusion problems with moving boundaries In Moving boundary problems (eds) D G Wilson (New York: Academic Press)

Shamsunder N, Sparrow E M 1975 Analysis of conduction phase-change via enthalpy model. Trans. ASME, J. Heat Transfer 97: 333-340

Sparrow E M, Schneider G E, Pletcher R H 1988 Handbook of numerical heat transfer (New York: Wiley) chap. 18

Szekely J, Themlis N J 1970 Rate phenomena in process metallurgy (New York: Wiley Interscience) chap. 10

Voller V R 1990 Fast implicit finite difference method for the analysis of phase-change problems. Numer. Heat Transfer B17: 155-169 
Voller V R, Cross M, Markatos N C 1987 An enthalpy method for convection diffusion phase change. Int. J. Numer. Methods Eng. 24: 271-284

Voller V R, Cross M, Walton P G 1979 Assessment of weak solution numerical techniques for solving Stefan problems. In Numerical methods in thermal problems (eds) R W Lewis, K Morgan (London: Pineridge)

Wilson D G, Soloman A D, Boggs P T 1978 Moving boundary problems (New York: Academic Press) 